\title{
BMJ Open Provider variability in the intraoperative use of neuromuscular blocking agents: a retrospective multicentre cohort study
}

\author{
Friederike C Althoff, ${ }^{1}$ Xinling Xu, ${ }^{1}$ Luca J Wachtendorf (D) , ${ }^{1}$ Denys Shay, ${ }^{1}$ \\ Maria Patrocinio, ${ }^{1}$ Maximilian S Schaefer, ${ }^{1,2}$ Timothy T Houle, ${ }^{3}$ \\ Philipp Fassbender, ${ }^{1,4}$ Matthias Eikermann (D) , Karuna Wongtangman (D) 1,6
}

To cite: Althoff FC, Xu X, Wachtendorf LJ, et al. Provider variability in the intraoperative use of neuromuscular blocking agents: a retrospective multicentre cohort study. BMJ Open 2021;11:e048509. doi:10.1136/ bmjopen-2020-048509

- Prepublication history and additional supplemental materials for this paper is available online. To view these files, please visit the journal online (http://dx.doi.org/10. 1136/bmjopen-2020-048509).

Received 29 December 2020 Revised 15 March 2021 Accepted 24 March 2021
Check for updates

(C) Author(s) (or their employer(s)) 2021. Re-use permitted under CC BY-NC. No commercial re-use. See rights and permissions. Published by BMJ.

For numbered affiliations see end of article.

Correspondence to Dr Matthias Eikermann; meikermann@montefiore.org

\section{ABSTRACT}

Objective To assess variability in the intraoperative use of non-depolarising neuromuscular blocking agents (NMBAs) across individual anaesthesia providers, surgeons and hospitals.

Design Retrospective observational cohort study. Setting Two major tertiary referral centres, Boston, Massachusetts, USA.

Participants 265537 adult participants undergoing noncardiac surgery between 0ctober 2005 and September 2017.

Main outcome measures We analysed the variances in NMBA use across 958 anaesthesia and 623 surgical providers, across anaesthesia provider types (anaesthesia residents, certified registered nurse anaesthetists, attendings) and across hospitals using multivariableadjusted mixed effects logistic regression. Intraclass correlations (ICC) were calculated to further quantify the variability in NMBA use that was unexplained by other covariates. Procedure-specific subgroup analyses were performed.

Results NMBAs were used in 183242 (69\%) surgical cases. Variances in NMBA use were significantly higher among individual surgeons than among anaesthesia providers (variance $1.32(95 \% \mathrm{Cl} 1.06$ to 1.60$)$ vs 0.24 (95\% Cl 0.19 to 0.28 ), $p<0.001$ ). Procedure-specific subgroup analysis of hernia repairs, spine surgeries and mastectomies confirmed our findings: the total variance in NMBA use that was unexplained by the covariate model was higher for surgeons versus anaesthesia providers (ICC $37.0 \%$ vs $13.0 \%, 69.7 \%$ vs $25.5 \%, 69.8 \%$ vs $19.5 \%$, respectively; $p<0.001)$. Variances in NMBA use were also partially explained by the anaesthesia provider's hospital network (Massachusetts General Hospital: variance 0.35 (95\% Cl 0.27 to 0.43 ) vs Beth Israel Deaconess Medical Center: 0.15 (95\% Cl 0.12 to 0.19$) ; p<0.001)$. Across provider types, surgeons showed the highest variance, and anaesthesia residents showed the lowest variance in NMBA use.

Conclusions There is wide variability across individual surgeons and anaesthesia providers and institutions in the use of NMBAs, which could not sufficiently be explained by a large number of patient-related and procedure-related characteristics, but may instead be driven by preference. Surgeons may have a stronger influence on a key aspect of anaesthesia management than anticipated.

\section{Strengths and limitations of this study}

- A large population of 265537 surgical patient cases with 958 anaesthesia and 623 surgical providers across two hospital networks was included, resulting in an opportunity to study provider variability across healthcare systems.

- The differential effects of stakeholders of different professions (anaesthetists, nurses and surgeons) on neuromuscular blocking agent utilisation could be examined and compared.

- Bias related to unmeasured confounding may arise due to the observational study design of this study.

- This study does not allow conclusions regarding potential relationships between provider variability and associated adverse outcomes such as surgical complications or implications on costs of care.

\section{INTRODUCTION}

Each year, several million patients worldwide receive general anaesthesia (GA) and mechanical ventilation for surgery. ${ }^{1}$ Neuromuscular blocking agents (NMBAs) are frequently administered to facilitate endotracheal intubation and mechanical ventilation in patients undergoing surgery and are known to optimise surgical conditions while achieving lower doses of volatile or intravenous anaesthetics. ${ }^{2}$ The use of NMBAs has been associated with a higher risk of postoperative respiratory complications, ${ }^{3-7}$ an increased risk of readmission to the hospital, ${ }^{8-10}$ higher risks of adverse postoperative cardiovascular events and decreased physical activity. ${ }^{11}$ Results of these studies further suggested a dose-dependent risk increase of postoperative complications after use of NMBAs. ${ }^{8-1012}$ In addition, a higher risk of postoperative residual neuromuscular blockade has been described if higher NMBA doses were administered. ${ }^{1}$ Residual neuromuscular blockade, in turn, is a risk factor of desaturation and reintubation and therefore may contribute to impaired recovery from GA. ${ }^{3}$ 
The increasing recognition of complications associated with the use of NMBAs has put into question the role of each physician in providing patient care. There is clinical evidence for the overuse of NMBAs. ${ }^{11}$ Possible explanations of this observation have not yet been studied. An indicator of overutilisation may be high provider variability that cannot be explained by various patient characteristics and procedural risk factors associated with NMBA use. Instead, we argue it might be driven by individual provider preference and hospital culture. ${ }^{13} \mathrm{~A}$ high provider variability, therefore, may contribute to adverse postoperative outcomes.

In this retrospective multicentre cohort study, we hypothesised that significant provider variability in the use of NMBAs exists across individual anaesthesiologists, surgeons and hospitals, indicating overutilisation and a lack of standardisation.

\section{METHODS}

\section{Study design and setting}

This retrospective multicentre cohort study was conducted at Massachusetts General Hospital (MGH) and Beth Israel Deaconess Medical Center (BIDMC), Massachusetts, USA. Data were obtained for patients undergoing non-cardiac surgery under GA between 1 October 2005 and 30 September 2017. Data were collected from hospital registry databases (online supplemental file 1, section 1). This manuscript adheres to the Strengthening the Reporting of Observational Studies in Epidemiology guidelines (online supplemental file 2). ${ }^{14}$

\section{Study cohort}

We included anaesthesia providers and surgeons who performed a minimum of 50 cases during the study period. Anaesthesia providers were either anaesthesia residents, certified registered nurse anaesthetists (CRNAs) or attending anaesthesiologists. Details on the designation of the primary anaesthesia provider for each case are provided in the online supplemental file 1 , section 1 .

Adult patients who underwent non-cardiac surgery with GA were eligible for inclusion in this study. Patients with an American Society of Anesthesiologists (ASA) physical status $>$ IV, those who underwent any surgery within 4 weeks prior to the index procedure or those with missing data for the primary analysis were excluded. The complete case method was used for the primary analysis.

\section{Primary analysis}

NMBA use was defined as intraoperative administration of any dose of atracurium, cisatracurium, rocuronium, pancuronium, mivacurium or vecuronium. In the primary analysis, we applied a mixed effects logistic regression model that included the use of NMBAs as the dependent variable and individual anaesthesia and surgical providers as two crossed random effects within the same model to assess the association between individual anaesthesia providers and surgeons and the use of NMBAs during
GA. ${ }^{15}$ First, a propensity score was generated to predict each patient's probability of receiving NMBAs adjusted for all covariates described below, as previously reported ${ }^{16}$ (online supplemental file 1, sections 1 and 2, table S1). The centred propensity score was then included as a continuous variable in the primary model, allowing for the individual intercept of a provider to be interpreted as the predicted probability that an average patient would receive NMBAs during surgery. An average patient was mathematically defined as a patient with an average probability of receiving NMBAs. From that model, we estimated the random effect variances for both random effects of anaesthesia providers and surgeons, respectively, and tested whether these variances were significantly different using empirical $p$ values based on posterior samples from our mixed effects model under the Bayesian framework. Finally, we used the random effect variances and the model residuals to calculate the intraclass correlation (ICC), thereby determining the approximate percentage of variance in NMBA use that could not sufficiently be explained by the covariates of the model and was therefore attributed to anaesthesia and surgical providers, respectively (online supplemental file 1 , section 2 ).

\section{Sensitivity analyses}

Several sensitivity and subgroup analyses were performed to assess the robustness of the primary analysis. To address potential concerns that the type of performed procedures may have differed among providers, we performed analyses in more homogenous subgroups: general surgery as well as procedure-specific subgroups of hernia repair surgeries, spine surgeries and mastectomies, respectively (online supplemental table S2). In addition, we tested the primary hypothesis when considering different provider's experience levels. Details on sensitivity analyses are provided in the online supplemental file 1, section 3 .

\section{Exploratory analyses}

To further explore a nested relationship between providers and hospitals, we determined variances in NMBA use across providers of the two major hospital networks included in this study (institutional effect). Therefore, we extended the primary model by adding the hospital network as a random effect to the model, nesting anaesthesia and surgical providers in their respective hospitals. In addition, we analysed whether differences in NMBA use across groups of different provider types (anaesthesia residents, CRNAs, anaesthesia attendings and surgeons) existed by adding the provider type as a random effect, nesting providers in provider types. Finally, we added the surgical specialty as a random effect where teams of anaesthesia providers and surgeons are typically more permanent, nesting anaesthesia and surgical providers in surgical specialties.

With an exploratory intent, we further investigated whether NMBA use facilitated the surgical condition and reduced surgical as well as anaesthetic time in patients who underwent hernia repair surgeries. We also assessed 
the variability among anaesthesia and surgical providers as well as across hospitals in the use of neostigmine for reversal of neuromuscular blockade. Lastly, we evaluated the provider variability in the use of different NMBA doses. Details on exploratory analyses are provided in the online supplemental file 1 , section 4 .

\section{Covariates}

We adjusted our analyses for a priori defined patient, procedure and anaesthesia-related covariates that could have affected the need for NMBAs and therefore the use and dose of NMBAs during surgery based on clinical plausibility and literature review. ${ }^{81217}$ We included baseline patient characteristics such as age, sex, body mass index, ASA physical status classification and Charlson Comorbidity Index. Procedure-related characteristics comprised duration of surgery, year of surgery, surgical specialty, admission type (ambulatory, same-day admission and inpatient), emergency surgery, night surgery (surgery between 17:00 and 07:00), ${ }^{18}$ handover between anaesthesia providers and work relative value units as a marker of surgical complexity. Within the subgroups of general surgery and hernia repairs, we additionally adjusted for the surgical approach (laparoscopic vs open, online supplemental table S3). ${ }^{19}$ To adjust for anaesthesia-related characteristics, we included depth of anaesthesia (age-adjusted minimum alveolar concentration of volatiles and nitrous oxide), intraoperative hypotension (defined as mean arterial pressure $<55 \mathrm{~mm} \mathrm{Hg}$ ), intraoperative cumulative doses of long-acting opioids, propofol, vasopressors, total intravenous administered amount of fluids, units of packed red blood cells, use of epidural anaesthesia and whether or not a patient was extubated before end of anaesthesia care. Online supplemental table S1 shows detailed information on all a priori defined covariates.

\section{Statistical analyses}

Covariates demonstrating linear associations with the outcome were included as continuous variables into the regression models. Variables with a non-linear relationship were categorised into quintiles or clinically relevant groups (online supplemental file 1, section 2). Unless otherwise specified, results of adjusted analyses are presented as random effect variances with $95 \%$ CIs. A two-sided $p$ value $<0.05$ was considered statistically significant. Analyses were performed in Stata V.13.0 (StataCorp) and RStudio V.3.2.5 (RStudio, package 'MCMCglmm'). ${ }^{20}$

\section{Patient and public involvement}

This was a retrospective cohort study, and no patients were involved in the study design, setting the research questions or the outcome measures directly. No patients were asked for advice on interpretation of results or writing this manuscript.
Patients aged $\geq 18$ who underwent non-cardiac surgery with general anaesthesia and were extubated post-procedure $\mathbf{n}=\mathbf{3 1 5} 503$

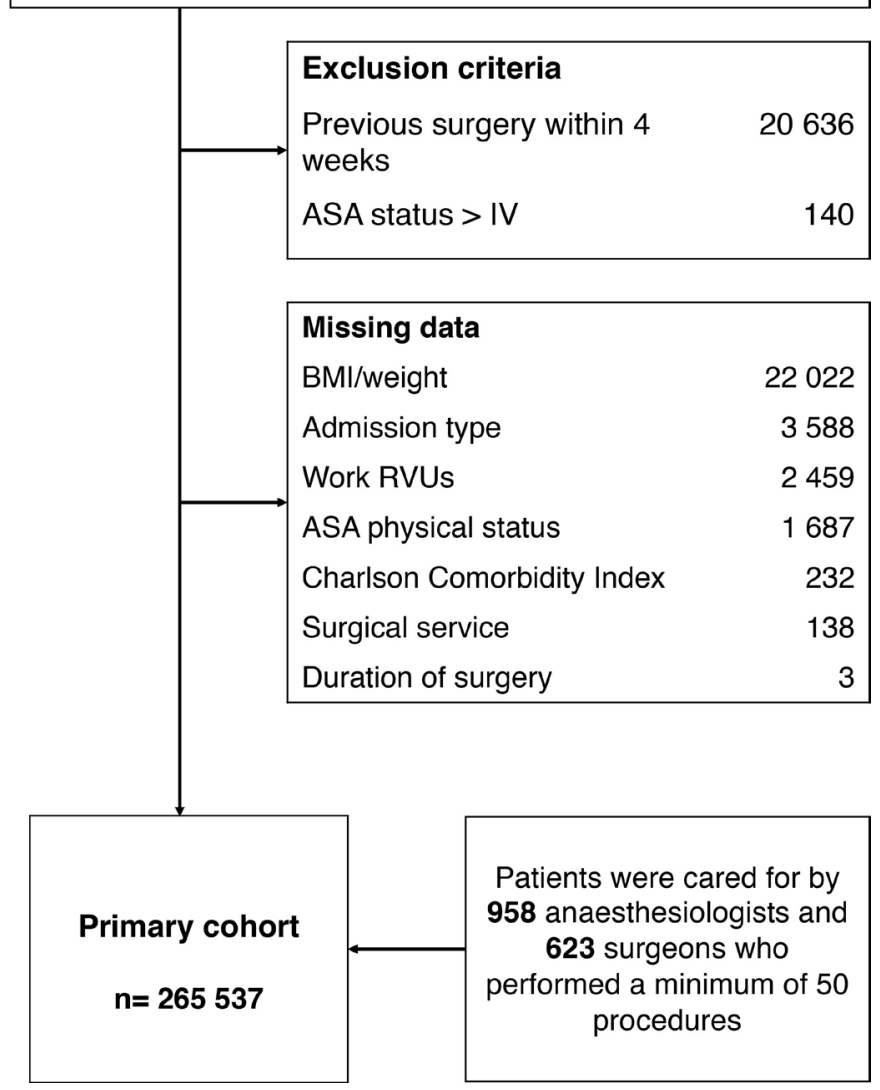

Figure 1 Study flow. Multiple exclusions may apply. ASA, American Society of Anesthesiologists; BMI, body mass index; RVUs, relative value units.

\section{RESULTS}

\section{Study cohort}

A total of 315503 patients were screened for eligibility. After application of exclusion criteria and exclusion due to missing data, the primary cohort consisted of 265537 patients who underwent surgery at either MGH $(\mathrm{n}=119393)$ or BIDMC $(\mathrm{n}=146144)$ (figure 1$)$. Intraoperative care was provided by 958 anaesthesia providers and 623 surgeons. The median number of cases per anaesthesia provider and surgeon was 618 (IQR 376, 909) and 1499 (IQR 716, 2604), respectively. NMBAs were administered in $69 \%$ of surgeries $(\mathrm{n}=183242)$. Table 1 displays patient characteristics and the distribution of variables by the use of NMBAs.

\section{Primary analysis}

The adjusted mean predicted probabilities for the intraoperative use of NMBAs during GA ranged from 1.4\% to $99.7 \%$ across individual surgeons and from $44.8 \%$ to $97.0 \%$ across individual anaesthesia providers. Random effect variances were calculated, and ICC was performed to quantify the interprovider variability in NMBA use that was unexplained by the covariates: across individual providers, surgeons had a significantly higher variance 
Table 1 Characteristics of the primary study cohort

\section{Primary study cohort \\ $\mathrm{n}=265537$}

\section{No NMBA use \\ ( $n=82$ 295)}

\section{Patient characteristics}

Age (years)

Sex, female

BMI $\left(\mathrm{kg} / \mathrm{m}^{2}\right)$

Charlson Comorbidity Index

ASA class

Emergency status

Admission type

Ambulatory
Same-day admission
Inpatient

\section{Surgical specialty}

Non-operating room anaesthesia

Burn surgery

Emergent-urgent surgical service

General surgery

Gynaecology

Neurosurgery

Oral/maxillofacial surgery

Orthopaedic surgery

Other

Otolaryngology

Paediatric surgery

Plastic surgery

Radiology

Surgical oncology

Thoracic surgery

Transplant surgery

Urology

Vascular surgery

\section{Intraoperative data}

Duration of surgery (min)

Work RVUs

Multiples of 95\% effective NMBA dose (ED95)

Steroidal (vs benzylisoquinolines) NMBAs*

End-tidal MAC of volatiles and nitrous oxide, age adjusted

Norepinephrine equivalent of vasopressor dose $(\mathrm{mg})$

PRBC units transfused

\begin{tabular}{lll}
\hline unit & $81952(99.6 \%)$ & $177958(97.1 \%)$ \\
$1-2$ units & $311(0.4 \%)$ & $4376(2.4 \%)$ \\
$\geq 3$ units & $32(<1 \%)$ & $908(0.5 \%)$ \\
Oral morphine equivalent of total opioid dose $(\mathrm{mg})$ & $0(0,6.80)$ & $12.00(0,23.80)$ \\
Overall amount of fluids $(\mathrm{L})$ & $1.5(1.0,2.5)$ & $2.3(1.3,3.5)$ \\
Total propofol dose $(\mathrm{mg})$ & $200(160,280)$ & $200(150,250)$ \\
\hline
\end{tabular}

Continued
NMBA use

$(n=183242)$

$\begin{array}{ll}51.99 \pm 16.71 & 54.40 \pm 16.45 \\ 45832(55.7 \%) & 104422(57.0 \%) \\ 27.72 \pm 6.31 & 28.58 \pm 6.96 \\ 0(0,2) & 1(0,3) \\ 2(2,2) & 2(2,3) \\ 2002(2.4 \%) & 7531(4.1 \%)\end{array}$

$56461(68.6 \%)$

$46379(25.3 \%)$

$18133(22.0 \%)$

$110553(60.3 \%)$

$7701(9.4 \%)$

$26310(14.4 \%)$

\begin{tabular}{ll}
\hline $2547(3.1 \%)$ & $2436(1.3 \%)$ \\
\hline $415(0.5 \%)$ & $926(0.5 \%)$ \\
$920(1.1 \%)$ & $6952(3.8 \%)$ \\
$9992(12.1 \%)$ & $37668(20.6 \%)$ \\
$8161(9.9 \%)$ & $20076(11.0 \%)$ \\
$2631(3.2 \%)$ & $14664(8.0 \%)$ \\
$805(1.0 \%)$ & $2142(1.2 \%)$ \\
\hline $24859(30.2 \%)$ & $35709(19.5 \%)$ \\
$1627(2.0 \%)$ & $931(0.5 \%)$ \\
\hline $4079(5.0 \%)$ & $3457(1.9 \%)$ \\
$152(0.2 \%)$ & $470(0.3 \%)$ \\
\hline $3919(4.8 \%)$ & $12657(6.9 \%)$ \\
$497(0.6 \%)$ & $630(0.3 \%)$ \\
$6683(8.1 \%)$ & $6491(3.5 \%)$ \\
$4452(5.4 \%)$ & $11542(6.3 \%)$ \\
\hline $457(0.6 \%)$ & $4164(2.3 \%)$ \\
\hline $8677(10.5 \%)$ & $14507(7.9 \%)$ \\
\hline $1422(1.7 \%)$ & $7820(4.3 \%)$
\end{tabular}

$82(57,123)$

$157(109,229)$

$7.30(4.74,12.13)$

$15.84(10.47,22.59)$

- $\quad 2.52(1.79,3.66)$

- $142397(77.7 \%)$

$0.91 \pm 1.55 \quad 0.94 \pm 0.33$

$0(0,0.02) \quad 0.01(0,0.15)$ 
Table 1 Continued

\begin{tabular}{|c|c|c|}
\hline $\begin{array}{l}\text { Primary study cohort } \\
\mathrm{n}=265537\end{array}$ & $\begin{array}{l}\text { No NMBA use } \\
(n=82 \text { 295) }\end{array}$ & $\begin{array}{l}\text { NMBA use } \\
(n=183242)\end{array}$ \\
\hline Total neostigmine dose (mg) & $0(0,0)$ & $3(2,4)$ \\
\hline Intraoperative hypotensive minutes of MAP $<55$ mm Hg (min) & $0(0,2)$ & $0(0,2)$ \\
\hline Out-of-hours surgery (17:00-07:00) & $4239(5.2 \%)$ & $11137(6.1 \%)$ \\
\hline \multicolumn{3}{|l|}{ Hospital } \\
\hline Massachusetts General Hospital & $25492(21.4 \%)$ & $93901(78.6 \%)$ \\
\hline Beth Israel Deaconess Medical Center & $56803(38.9 \%)$ & $89341(61.1 \%)$ \\
\hline Handover of anaesthesia care & $5133(6.2 \%)$ & $22217(12.1 \%)$ \\
\hline \multicolumn{3}{|l|}{ Anaesthesia provider types: number of cases (\%) performed by } \\
\hline
\end{tabular}

Normally distributed continuous variables were expressed as mean $( \pm \mathrm{SD})$, non-normally distributed variables as median (IQR) and categorical variables as frequency (percentages).

*Steroidal NMBAs include vecuronium, rocuronium and pancuronium. Benzylisoquinolines include cisatracurium, atracurium and mivacurium. ASA, American Society of Anesthesiologists physical status classification system; BMI, body mass index; CRNA, certified registered nurse anaesthetist; MAC, minimum alveolar concentration; MAP, mean arterial pressure; NMBA, neuromuscular blocking agent; PRBC, packed red blood cells; RVU, relative value units.

for NMBA use than anaesthesia providers (variance 1.32 (95\% CI 1.06 to 1.60 ) vs 0.24 (95\% CI 0.19 to 0.28 ); $\mathrm{p}<0.001$; figure 2A). ICC demonstrated that surgeons accounted for approximately $56.5 \%$, and anaesthesia providers accounted for approximately $10.2 \%$ of the total variance in NMBA use. Detailed results are provided in online supplemental table S4.

\section{Sensitivity analyses}

The primary findings remained robust throughout several sensitivity analyses. Forty-seven thousand six hundred and sixty patients underwent general surgery performed by 134 surgeons with anaesthesia care by 950 individual anaesthesia providers. The results of the primary analysis remained robust across individual providers, with even higher differences in variances in NMBA use between individual surgeons compared with anaesthesia providers (variance 2.96 (95\% CI 2.01 to 4.10 ) vs 0.26 (95\% CI 0.20 to 0.32 ); ICC $87.4 \%$ vs $7.6 \%$; $\mathrm{p}<0.001$ ). We further analysed procedure-specific subgroups of 12077 patients who underwent hernia repair surgeries, 13797 patients who underwent spine surgeries and 6936 patients who underwent mastectomies and breast reconstruction surgeries. We found that there is also wide variability in NMBA use even within the procedure-specific subgroups (figure 2B,C). Individual surgeons showed a significantly higher variance in NMBA use than anaesthesia providers across all subgroups and sensitivity analyses, including adjustment for differences in an individual provider's experience level (online supplemental file 1, section 3, tables S4 and S5).

\section{Exploratory analyses}

Variability across hospitals

Across hospitals, the use of NMBAs among all patient cases was $15.8 \%$ higher at MGH than at BIDMC ( $<<0.001$ in a two-sample test of proportions). In adjusted analysis, anaesthesia providers at MGH showed significantly higher variances in the use of NMBAs than at BIDMC (variance 0.35 (95\% CI 0.27 to 0.43 ) vs 0.15 (95\% CI 0.12 to 0.19 ), $\mathrm{p}<0.001)$. However, no significantly different variances in the use of NMBAs between surgeons of the two hospitals were observed (variance 1.30 (95\% CI 0.98 to 1.61 ) vs 1.36 (95\% CI 1.00 to 1.71$), \mathrm{p}=0.715$, online supplemental figure S1). ICC results across the two hospitals are displayed in online supplemental table S4.

\section{Variability across provider types}

Overall, the highest variance in NMBA use was seen for surgeons (variance $1.29,95 \%$ CI 1.05 to 1.55 ). Across different anaesthesia provider types, CRNAs tended to show the highest variance (variance $0.49,95 \%$ CI 0.35 to 0.66 ), followed by attendings (variance $0.45,95 \%$ CI 0.34 to 0.56 ). The lowest variance was observed for residents (variance $0.13,95 \%$ CI 0.10 to 0.16 ; online supplemental figure S2). However, variances in NMBA use were only significantly different when comparing anaesthesia residents $(\mathrm{p}<0.001)$ and surgeons $(\mathrm{p}<0.001)$ to all provider types, respectively. There was no significant difference between CRNAs and attendings. ICC results across hospitals are displayed in online supplemental table S4. 
Across surgical fields

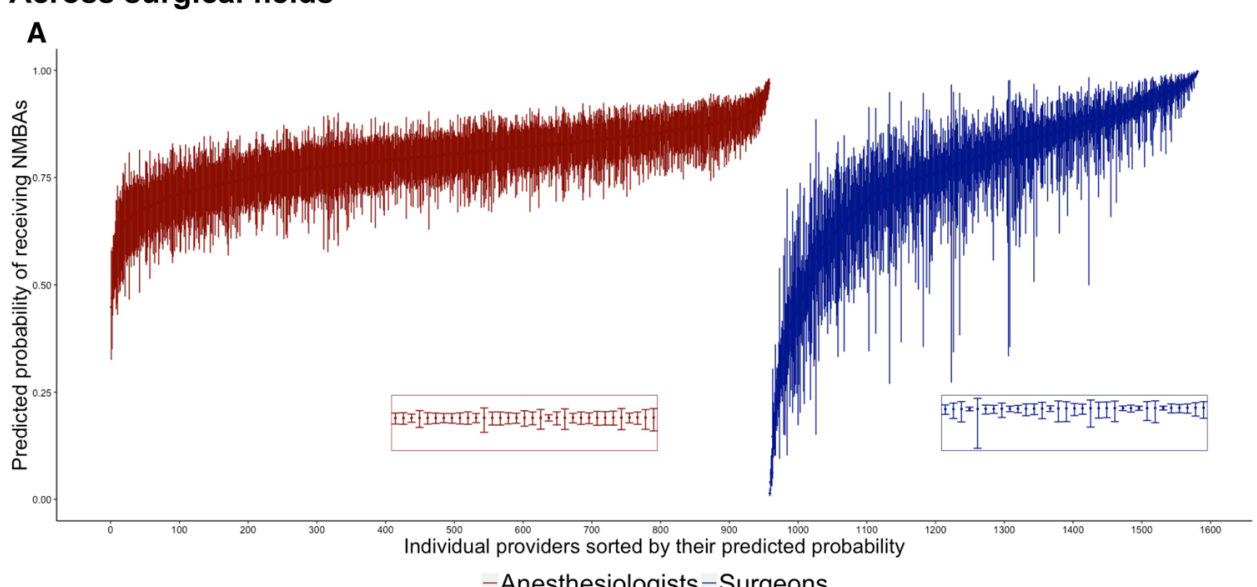

-Anesthesiologists-Surgeons

Procedure-specific subgroup of hernia repair surgery

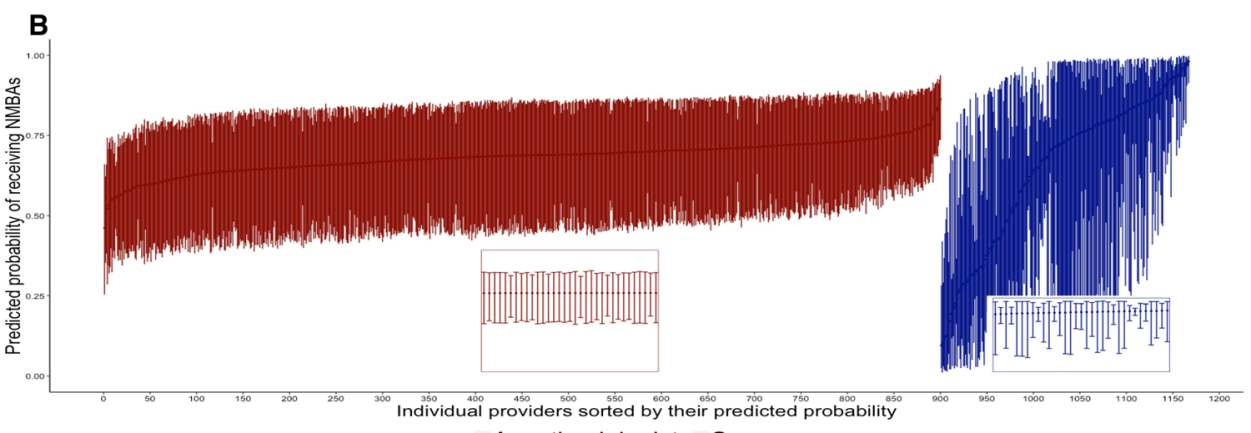

-Anesthesiologists-Surgeons

Procedure-specific subgroup of spine surgery

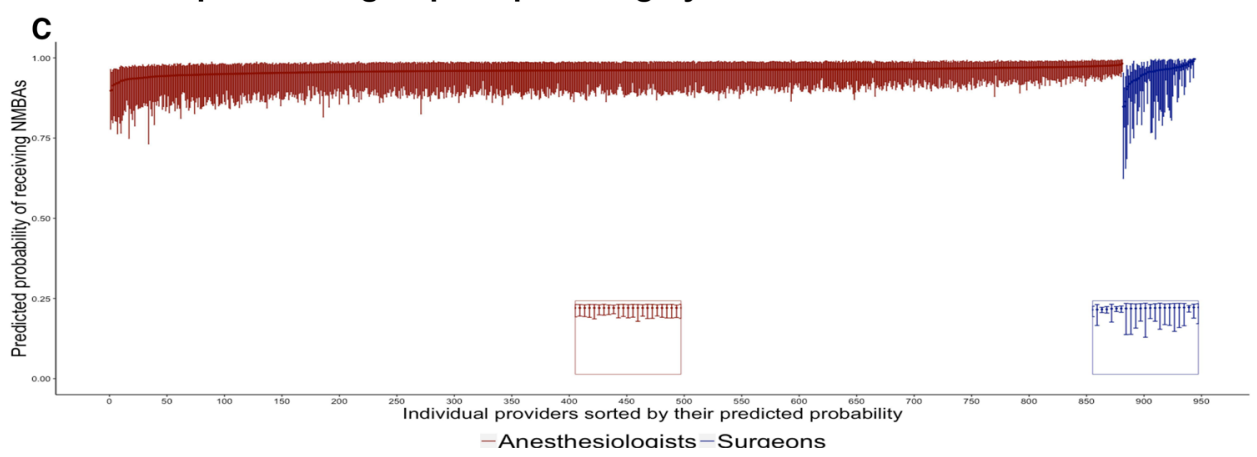

Figure 2 Provider variability in neuromuscular blocking agent (NMBA) use across anaesthesiologists and surgeons. The range of predicted probabilities for the use of NMBAs (mean and $95 \% \mathrm{Cl}$ ) across individual providers was obtained from the primary adjusted mixed effects model. Zooms show individual anaesthesia providers or surgeons in detail such that mean and $95 \%$ $\mathrm{Cl}$ become visible. Across surgical fields (A) and within procedure-specific subgroups, (B) hernia repair surgeries and (C) spine surgeries, respectively, surgeons accounted for a significantly higher amount of the total variance in NMBA use. This variance could not sufficiently be explained by the covariate model accounting for patient, procedure and anaesthesia-related factors that could have affected the use of NMBAs during surgery.

Variability across surgical specialties

When testing a nested relationship between providers and surgical specialties where teams of surgeons and anaesthesia providers are typically more permanent, surgeons showed a significantly higher variance in NMBA use than anaesthesia providers in the surgical specialties of general surgery (ICC $84.4 \%$ vs 5.0\%), neurosurgery (ICC 59.6\% vs $7.8 \%$ ), orthopaedic surgery (ICC $50.7 \%$ vs $16.3 \%$ ), otolaryngology (ICC $77.4 \%$ vs $13.5 \%$ ), surgical oncology (ICC $82.8 \%$ vs $7.5 \%$ ) and thoracic surgery (ICC $81.6 \%$ vs $3.8 \%$ ) (online supplemental table S4).
Effect of NMBA use on surgical and anaesthetic duration in hernia repair surgeries

The use of NMBAs in hernia repair surgeries was associated with a longer anaesthetic duration $(112(87,154)$ vs $89(69,110) \mathrm{min}$, adjusted incidence rate ratio (aIRR) $1.13,95 \%$ CI 1.10 to 1.17 , adjusted absolute difference $14.5 \mathrm{~min}, \mathrm{p}<0.001)$ and a longer surgical time $(78.6$ (56.8, $118.0)$ vs $61.2(43.7,80.8) \mathrm{min}$, aIRR $1.13,95 \%$ CI 1.09 to 1.18 , adjusted absolute difference $10.6 \mathrm{~min}, \mathrm{p}<0.001$ ). These differences in anaesthetic and surgical duration if NMBAs were used were not significantly modified by the 
surgical approach (laparoscopic vs open; p for interaction $=0.138$ for anaesthetic duration and 0.362 for surgical duration, respectively). Details are provided in the online supplemental file 1 , section 4 .

\section{Variability in the use of neostigmine for reversal}

Neostigmine was administered in $55.3 \%$ of the included surgical cases (146 849/265 537). Individual anaesthesia providers showed a significantly higher variance in neostigmine use than individual surgeons (variance 0.57 (95\% CI 0.52 to 0.64 ) vs 0.18 (95\% CI 0.15 to 0.20 ); ICC $56.3 \%$ vs $17.3 \%$; $<0.001)$. Across anaesthesia provider types, the highest variance was observed for attendings (variance $1.17,95 \%$ CI 0.89 to 1.46 ), followed by CRNAs (variance $0.85,95 \%$ CI 0.65 to 1.08 ). Residents showed the lowest variance (variance $0.40,95 \%$ CI 0.34 to 0.47 ). We did not find significant differences in variance in neostigmine use across hospitals (online supplemental table S6).

\section{Variability in NMBA dose}

One hundred eighty-three thousand two hundred and forty-two patient cases with intraoperative use of NMBAs were included in this analysis. Variances in administered NMBA ED95 total dose tended to be slightly higher across individual surgeons compared with anaesthesia providers. However, this difference was not statistically significant $(\mathrm{p}=0.18$; online supplemental table $\mathrm{S} 7)$.

\section{DISCUSSION}

This large multicentre study provides the first analysis to date demonstrating substantial provider variability in the use of NMBAs during GA. Among 1581 providers delivering care for more than 260000 surgical patients, our data suggest a high variance in NMBA use which might be explained by individual provider preferences. This variability in practice pattern could not sufficiently be explained by a large number of patient-related and procedure-related characteristics and risk factors that could have influenced the need for NMBAs. In addition, we demonstrate that surgeons showed a significantly higher proportion of the total variance in intraoperative NMBA use than anaesthesia providers.

We observed that variability in the use of NMBAs differed across different provider types and hospitals. Surgeons, in particular, showed the highest providerspecific variability in the use of NMBAs. Within the same surgical specialty, some surgeons only had NMBA administrations in $1.4 \%$ of their cases, and others were characterised by NMBA administration rates by up to 99.7\% of their cases under GA. Anaesthesia providerattributable preferences for NMBA varied less compared with surgeons, with predicted probabilities for the use of NMBAs ranging from $44.8 \%$ to $97.0 \%$ across each individual anaesthesia provider. The high variability in surgeon-attributable utilisation of NMBAs remained high in procedure-specific subgroups of patients undergoing general surgery, hernia repair surgeries, spine surgeries, as well as mastectomies. Based on our data, we hypothesise that variability among surgeons is driven primarily by the thought process that deeper levels of neuromuscular blockade are necessary for adequate operating conditions. ${ }^{17} 2122$ This is supported by our finding that the provider-related variability of neostigmine use could only be less explained by the surgeon but by the anesthesia provider (17.3\% vs $56.3 \%$, respectively) as this drug does not affect surgical conditions and is administered towards the end of a case.

Surgeons may overemphasise the need for NMBAs to achieve optimal surgical conditions. ${ }^{23}$ In fact, excellent surgical conditions can be achieved without NMBAs, and this practice may help improve operating room efficiency in some clinical scenarios. ${ }^{24}$ Our data showed a longer duration of surgery in the subgroup of patients undergoing hernia repair surgeries if NMBAs were administered. Recently, we demonstrated that the use of a laryngeal mask airway compared with endotracheal intubation helps decrease the risk of postoperative respiratory complications. ${ }^{25}$ These data support the view that anaesthesia and surgical providers should create interprofessional standardised guidelines for the use of NMBAs and airway devices to help increase patient safety and clinical effectiveness.

The use of NMBAs has long been deeply ingrained in standard anaesthetic management and therefore challenging this dogma is heavily subject to variations, as noted in our study. Variability in the use of neuromuscular blockade may reflect overutilisation of NMBAs in some patients and procedures, which in turn can lead to postoperative complications and unnecessary utilisation of healthcare resources. ${ }^{3-11}$ Given our findings of high provider variability across surgical fields and within subgroups, optimal doses of NMBAs should be determined in a patient characteristic-specific and procedurespecific fashion to provide a standardised guidance in the rational use of NMBAs.

Among anaesthesia providers, variances in the use of NMBA tended to be the highest for CRNAs. Based on this suggested trend, we speculate that CRNAs may tailor the practice more specifically to each surgeon. The finding of variability across anaesthesia providers is striking, as it may indicate overconfidence in clinical decision-making in the absence of a literature-based review. A recently concluded international survey noted that despite years of clinical practice, anaesthesia providers remain overconfident in their ability to manage neuromuscular blockade and depend more on intuition than objective monitoring. ${ }^{26}$ This is likely a key driver of the variability in NMBA use that was noted in our study.

The use of NMBAs also differed across hospitals. In a multivariable-adjusted analysis, individual providers in the centre with overall higher NMBA use also showed higher interprovider variability in NMBA use compared with those providers in the centre with less NMBA use. These findings emphasise the importance of the impact 
of hospital culture on practice patterns and provide an additional avenue for change.

The methods used in this study have limitations. The retrospective design of the presented study may have prevented us from taking into account factors that may have influenced the use and necessity of NMBAs during surgery. It is possible that high provider variability in the use of NMBAs reflects different procedure distributions across providers, particularly surgeons. We performed several sensitivity analyses in procedure-specific subgroups of patients undergoing hernia repair surgeries, spine surgeries, mastectomies and surgical specialties, which revealed that individual surgeons still accounted for a higher percentage of the total variance NMBA use than anaesthesia providers. This finding was also significant when accounting for the surgical approach. However, considering the diversity of surgical procedures, surgeons may be more closely associated with their procedures than we studied. This study does not allow any conclusions regarding potential relationships between the observed provider variability and associated adverse outcomes such as surgical complications or implications on costs of care. However, provider variability in the use of NMBAs has not been objectively shown before. Therefore, we believe in adding important value to the discussion about standardised guidelines on the use of NMBAs.

\section{CONCLUSION}

We demonstrated substantial variability in the use of NMBAs across individual surgeons and anaesthesia providers and across hospitals. Given the risk associated with NMBAs, high variability across providers, indicating a liberal use, underscores the need for standardised guidelines in NMBA use. Surgeons may have a stronger influence on a key aspect of anaesthesia management than anticipated. Interprofessional education on optimal use of NMBAs may be warranted.

\section{Author affiliations}

${ }^{1}$ Department of Anesthesia, Critical Care and Pain Medicine, Beth Israel Deaconess Medical Center and Harvard Medical School, Boston, MA, USA

${ }^{2}$ Department of Anesthesiology, Duesseldorf University Hospital, Duesseldorf, Germany

${ }^{3}$ Department of Anesthesia, Critical Care and Pain Medicine, Massachusetts General Hospital and Harvard Medical School, Boston, MA, USA

${ }^{4}$ Klinik für Anästhesiologie, Operative Intensivmedizin, Schmerz- und

Palliativmedizin, Marien Hospital Herne, Universitätsklinikum der Ruhr-Universität Bochum, Bochum, Germany

${ }^{5}$ Department of Anesthesiology, Montefiore Medical Center and Albert Einstein

College of Medicine, Bronx, NY, USA

${ }^{6}$ Department of Anesthesiology, Faculty of Medicine, Siriraj Hospital, Mahidol University, Bangkok, Thailand

\section{Twitter Luca J Wachtendorf @WachtendorfLuca}

Contributors Study concept and design: FCA, ME, XX, MP. Acquisition, analysis or interpretation of data: All authors. Drafting of the manuscript: FCA, KW, XX, KW, MSS, PF, ME. Critical revision of the manuscript for important intellectual content: All authors. Statistical analysis: FCA, XX, KW, TTH, KW, DS, MP, ME. Obtained funding: ME. Administrative, technical or material support: All authors. Study supervision: $X X$, TTH, ME. The manuscript's guarantor is ME.
Funding This work was supported by Jeffrey and Judith Buzen in an unrestricted grant to ME (222302). ME received funding for investigator-initiated trials from Merck (agreement number 57669 US).

Competing interests TTH reports grants from NINDS (PI), grants from NIGMS, personal fees from Headache, personal fees from Anesthesiology, personal fees from Cephalalgia, outside the submitted work. ME received honorarium for giving advice to Merck, holds equity of Calabash Bioscience and is an Associate Editor of the British Journal of Anaesthesia.

Patient consent for publication Not required.

Ethics approval The Committee on Clinical Investigations affiliated with Beth Israel Deaconess Medical Center (protocol number 2017P000641) and the Partners Institutional Review Board (protocol number 2017P002631) at Massachusetts General Hospital approved the study. The requirement for informed consent was waived.

Provenance and peer review Not commissioned; externally peer reviewed.

Data availability statement Data are available upon reasonable request. The data sets analysed during the present study are not publicly available due to privacy issues pertaining to the study population. The material presented in this study has not been included in any other manuscript or published on the internet.

Supplemental material This content has been supplied by the author(s). It has not been vetted by BMJ Publishing Group Limited (BMJ) and may not have been peer-reviewed. Any opinions or recommendations discussed are solely those of the author(s) and are not endorsed by BMJ. BMJ disclaims all liability and responsibility arising from any reliance placed on the content. Where the content includes any translated material, BMJ does not warrant the accuracy and reliability of the translations (including but not limited to local regulations, clinical guidelines, terminology, drug names and drug dosages), and is not responsible for any error and/or omissions arising from translation and adaptation or otherwise.

Open access This is an open access article distributed in accordance with the Creative Commons Attribution Non Commercial (CC BY-NC 4.0) license, which permits others to distribute, remix, adapt, build upon this work non-commercially, and license their derivative works on different terms, provided the original work is properly cited, appropriate credit is given, any changes made indicated, and the use is non-commercial. See: http://creativecommons.org/licenses/by-nc/4.0/.

\section{ORCID iDs}

Luca J Wachtendorf http://orcid.org/0000-0002-9966-2092

Matthias Eikermann http://orcid.org/0000-0002-7893-0596

Karuna Wongtangman http://orcid.org/0000-0003-2444-8275

\section{REFERENCES}

1 Lien CA, Eikermann M. 22 - Neuromuscular blockers and reversal frugs. In: Hemmings HC, Egan TD, eds. Pharmacology and physiology for anesthesia. 2nd edn. Philadelphia: Elsevier, 2019: 428-54.

2 Boon M, Martini C, Dahan A. Recent advances in neuromuscular block during anesthesia. F1000Res 2018;7:167.

3 Berg H, Roed J, Viby-Mogensen J, et al. Residual neuromuscular block is a risk factor for postoperative pulmonary complications. A prospective, randomised, and blinded study of postoperative pulmonary complications after atracurium, vecuronium and pancuronium. Acta Anaesthesiol Scand 1997;41:1095-103.

4 Grabitz SD, Rajaratnam N, Chhagani K, et al. The effects of postoperative residual neuromuscular blockade on hospital costs and intensive care unit admission: a population-based cohort study. Anesth Analg 2019;128:1129-36.

5 Baillard C, Clec'h C, Catineau J, et al. Postoperative residual neuromuscular block: a survey of management. $\mathrm{Br} J$ Anaesth 2005;95:622-6.

6 Hayes AH, Mirakhur RK, Breslin DS, et al. Postoperative residual block after intermediate-acting neuromuscular blocking drugs. Anaesthesia 2001;56:312-8.

7 Murphy GS, Brull SJ. Residual neuromuscular block: lessons unlearned. Part I: definitions, incidence, and adverse physiologic effects of residual neuromuscular block. Anesth Analg 2010;111:120-8.

8 Thevathasan T, Shih SL, Safavi KC, et al. Association between intraoperative non-depolarising neuromuscular blocking agent dose and 30-day readmission after abdominal surgery. $\mathrm{Br} J$ Anaesth 2017;119:595-605. 
9 McLean DJ, Diaz-Gil D, Farhan HN, et al. Dose-dependent association between intermediate-acting neuromuscular-blocking agents and postoperative respiratory complications. Anesthesiology 2015;122:1201-13.

10 Kirmeier E, Eriksson LI, Lewald $\mathrm{H}$, et al. Post-anaesthesia pulmonary complications after use of muscle relaxants (popular): a multicentre, prospective observational study. Lancet Respir Med 2019;7:129-40.

11 National Heart, Lung, and Blood Institute PETAL Clinical Trials Network, Moss M, Huang DT, et al. Early neuromuscular blockade in the acute respiratory distress syndrome. $N$ Engl $\mathrm{J}$ Med 2019;380:1997-2008.

12 Scheffenbichler FT, Rudolph MI, Friedrich S, et al. Effects of high neuromuscular blocking agent dose on post-operative respiratory complications in infants and children. Acta Anaesthesiol Scand 2020;64:156-67.

13 Palsen S, Wu A, Beutler SS, et al. Investigation of intraoperative dosing patterns of neuromuscular blocking agents. J Clin Monit Comput 2019;33:455-62.

14 von Elm E, Altman DG, Egger M, et al. The strengthening the reporting of observational studies in epidemiology (STROBE) statement: guidelines for reporting observational studies. J Clin Epidemiol 2008;61:344-9.

15 Baayen RH, Davidson DJ, Bates DM. Mixed-effects modeling with crossed random effects for subjects and items. Journal of Memory and Language 2008;59:390-412.

16 Ladha KS, Bateman BT, Houle TT, et al. Variability in the use of protective mechanical ventilation during general anesthesia. Anesth Analg 2018;126:503-12.

17 King M, Sujirattanawimol N, Danielson DR, et al. Requirements for muscle relaxants during radical retropubic prostatectomy. Anesthesiology 2000;93:1392-7.
18 Althoff FC, Wachtendorf LJ, Rostin P, et al. Effects of night surgery on postoperative mortality and morbidity: a multicentre cohort study. BMJ Qual Saf 2020

19 Dexter F, Dexter EU, Ledolter J. Importance of appropriately modeling procedure and duration in logistic regression studies of perioperative morbidity and mortality. Anesthesia \& Analgesia $2011 ; 113$

20 Hadfield JD. MCMC methods for multi-response generalized linear mixed models: the MCMCgImm $R$ package. J Stat Softw 2010;33.

21 Madsen MV, Staehr-Rye AK, Gätke MR, et al. Neuromuscular blockade for optimising surgical conditions during abdominal and gynaecological surgery: a systematic review. Acta Anaesthesiol Scand 2015;59:1-16.

22 Fuchs-Buder T, Schmartz D, Baumann C, et al. Deep neuromuscular blockade improves surgical conditions during gastric bypass surgery for morbid obesity: a randomised controlled trial. Eur $J$ Anaesthesiol 2019;36:486-93.

23 YL L, Liu YL, CM X. The effects of neuromuscular blockade on operating conditions during general anesthesia for spinal surgery. J Neurosurg Anesthesiol 2014;26:45-9.

24 Alfille $\mathrm{PH}$, Merritt C, Chamberlin NL, et al. Control of perioperative muscle strength during ambulatory surgery. Curr Opin Anaesthesiol 2009;22:730-7.

25 Hammer M, Santer P, Schaefer MS, et al. Supraglottic airway device versus tracheal intubation and the risk of emergent postoperative intubation after general anaesthesia in adults: a retrospective cohort study. Br J Anaesth 2021;126:738-45.

26 Naguib M, Brull SJ, Hunter JM, et al. Anesthesiologists' Overconfidence in their perceived knowledge of neuromuscular monitoring and its relevance to all aspects of medical practice: an international survey. Anesth Analg 2019;128:1118-26. 